\title{
Traditionelle Chinesische Medizin (TCM)
}

\section{1. Übersicht}

\subsection{Was ist TCM (Begriffsbestimmung)}

Die Traditionelle Chinesische Medizin (TCM) ist der Oberbegriff für mehrere im Chinesischen Kulturkreis entstandenen und miteinander verbundene Therapieformen. Alle Verfahren der TCM beruhen auf (ganzheitlichen) Konzepten, in denen der Behandlungsschwerpunkt nicht auf den Symptomen, sondern auf der die Krankheit ermöglichenden Konstitutionsstörung (Disharmonie) liegt.

Ziel der Therapie mit Traditioneller Chinesischer Medizin ist die Normalisierung der den Lebensprozeß bestimmenden Fließgleichgewichte.

Eine symptomatische Behandlung und die Behandlung schwerwiegender Erkrankungen durch die "klassische westliche Medizin" wird durch die TCM nicht ausgeschlossen.

\subsection{Woher kommt TCM (Historisches)}

Die ersten heute noch bekannten Werke der Traditionellen Chinesischen Medizin stammen aus dem 4.-2. Jahrhundert v.Chr. Neben einem großen Erfahrungsschatz durch Naturbeobachtung (Daoistische Philosophie) sind verschiedene ineinander übergehende Theorieansätze aus der Philosophieschule des Konfuzius bestimmend. Nach einer Blütezeit im Mittelalter (ca.1000 - 1350 n.Chr.) entstanden verschiedene philosophische Schulen, die sich alle in der Praxis nicht durchsetzen konnten. Anfang dieses Jahrhunderts wurde die TCM durch die westliche Medizin massiv zurückgedrängt.

Das Wiederaufleben ist auf die Propagierung der TCM als Breiten- und Volksmedizin durch Mao Zedong in den 50er Jahren (Kulturrevolution) zurückzuführen.

Heute existieren beide Medinzinformen gleichberechtigt nebeneinander, jeder Arzt kennt beide Therapiemethoden: die Arztausbildung existiert in zwei Formen/Studiengängen, die 4 Jahre in der Schwerpunktrichtung und ein Jahr der Komlementärrichtung ausbilden.

\subsection{Welche Therapieformen gehören zu TCM (Formen)}

Akkupunktur

Stimulation des Organismus und seiner Funktionsbereiche durch Nadeln. Die an bestimmten Stellen in die Haut eingestochenen Nadeln stimulieren die mit diesem Akkupunkturpunkt verbundenen Funktionsbereiche bzw. Meridiane. Sie ist -entgegen verbreiteter Vorstellungen- nicht schmerzfrei. Die Akkupunktur gehört zu den milderen, äußeren Behandlungsformen.

Moxibustion

Akkupunktur durch Wärmereizung. Stammt wahrscheinlich aus dem kälteren Norden Chinas. Speziell aufbereitete Beifußkügelchen werden auf bestimmten Hautstellen abgebrannt und geben Ihre Wärme an den Körper ab. Durch die Doppeleigenschaft der Wärme als Reiz und als therapeutisches Prinzip ist eine Gleichsetzung mit der Akkupunktur nicht berechtigt.

Pharmakotherapie

Die Pharmakotherapie bildet den Schwerpunkt für die TCM, ca. 70-80\% aller TCM- 
Behandlungen in China werden über dieses Therapieprinzip vorgenommen. Dabei werden mineralische, tierische und pflanzliche Drogen eingesetzt, die pflanzlichen bestimmen aber mit über $80 \%$ das Gesamtbild. Die Pharmakotherapie ist eine hochwirksame, mehr die inneren, grundlegenderen Funktionsbereiche beeinflussende Therapieform.

Diätetik

Die Diätetik betrifft die gesamte Lebensweise, den Lebensstil: Den Nahrungsmittel werden, wie auch den Pharmaka und den Verhaltensweisen (z.B. Tagesrhythmus), verschiedene Wirkrichtungen zugeordnet. Damit ist es bei leichten Erkrankungen möglich, durch gezielte Veränderung des Lebensstiles (Diät) die gestörten Körpervorgänge wieder zu normalisieren.

Tui Na und An Mo

(Massagetherapien)

Massagetherapien beruhen auf den Prinzipien des Energieflusses und der Meridiane und bauen den Stau des Energieflusses ab.

Qui Gong und Taiji Quan

(Bewegungstherapien)

Beeinflussung der gestörten Funktionsabläufe durch Bewegung (z.B. mentale oder emotionale Beeinflussung, Streßabbau, Organtraining)

\section{Die Diagnostik}

Die Diagnostik der TCM beruht auf nichtinvasiven Methoden (Beobachten, Tasten/Fühlen, Fragen). Die Diagnostik ist sehr zeitaufwendig und recht schwer zu erlernen.

Ziel ist es, sich ein Bild vom Gesamtzustand des Patienten zu machen: Anamnese, die Haut- und Gesichtsfarbe, das Auftreten und die Erscheinung des Patienten und besonders die komplizierte Zungen- und Pulsdiagnose geben ein Gesamtbild, aus dem dann die Ursache der Erkrankung ableitbar ist.

\subsection{Zungendiagnostik}

Die Zunge wird nach einem umfangreichen Kriterienkatalog, der u.a. Belag, Dicke, Feuchtigkeitsgrad und Färbung umfaßt, untersucht.

Aus diesen Kriterien ist ein Rückschluß auf den funktionalen Status der einzelnen Grundorgane und Funktionsbereiche und dort vorliegende Störungen möglich.

\subsection{Pulsdiagnostik}

Der Puls wird an beiden Handgelenken an je 3 Positionen geprüft und nach 28 verschiedenen Pulsqualitäten beurteilt. Es gibt auch Zuordnungen der einzelnen Positionen zu den Hauptorganen und deren Leitbahnen, so daß indirekt Angaben über den ganzen Körper zugänglich sind. 


\section{Das Krankheitsbild der TCM (Theorie)}

\subsection{Yin und Yang}

Das wahrscheinlich älteste und universellste Konzept zur Erklärung der den Körperzustand bestimmenden Fließgleichgewichte ist die Yin-Yang-Theorie.

Ausgangsbasis bildet die universelle Polarität von Yin und Yang, zweier entgegengesetzter und doch einander bedingender Begriffe. Sie bilden eine dialektische Einheit, gehen auch ineinander über und lösen einander (rhythmisch) ab.

Es sind keine absoluten Kategorien: Wie aus dem Symbol erkannt werden kann, enthält jedes Prinzip auch Elemente des anderen.

Die Yin-Yang-Polarität ist durch drei Beziehungen gekennzeichnet: wechselseitige Abhängigkeit wechselseitige Begünstigung und Abschwächung wechselseitige Umwandlung

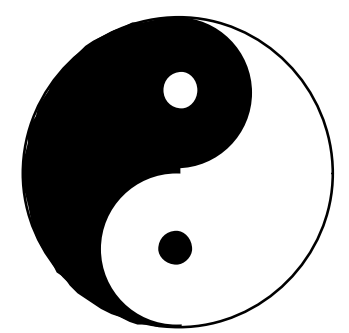

In der TCM steht Yin für das weibliche, passive, strukturbildende Prinzip und Yang für das männliche, aktive, bewegende Prinzip.

Unsere gesamte sichtbare Welt einschließlich unseres eigenen Körpers läßt sich in diese beiden Prinzipien einteilen. Die folgende Tabelle gibt eine Übersicht:

\begin{tabular}{|l|l|}
\hline Yang & Yin \\
\hline & \\
\hline männlich & Weiblich \\
\hline gebendes Prinzip & empfangendes Prinzip \\
\hline Sonne & Mond \\
\hline Bewußtes & Unbewußtes \\
\hline Ratio & Emotio \\
\hline Himmel & Erde \\
\hline Tag & Nacht \\
\hline Licht & Dunkelheit \\
\hline Hitze & Kälte \\
\hline Bewegung & Ruhe \\
\hline & \\
\hline Aktivität & Struktivität \\
\hline zusammenziehendes Prinzip & ausdehnendes Prinzip \\
\hline zuviel & Zuwenig \\
\hline körperlich: Überfunktion & körperlich: Unterfunktion \\
\hline
\end{tabular}

Achtung: Das gleiche Element kann je nach betrachtetem Aspekt/Eigenschaft einmal Yin und ein anderes Mal Yang sein 


\subsection{Die Wandlungsphasen (Orbis, Organe)}

Die Organe (Orbis, Wandlungsphasen) bilden funktionale Bereiche. Sie sind nicht mit dem westlichen Organbegriff vergleichbar, da z.B. keine räumliche Zuordnung getroffen wird.

Therapeutisch gebräuchlich sind 10 Grund- und 2 Sonderorgane mit unterschiedlichen Aufgaben. Sie werden durch das für die Akkupunktur wichtige Leitbahnen-System und die verschiedenen Elemente (Qi, Blut, Jing, Shen) ergänzt.

Das auf der Fünfzahl basierende Modell (2x5 Organe) ist vor allem in der westlichen "Sekundärliteratur" verbreitet. Es wird auch im Arzneibuch der Chin. Medizin verwendet, ist aber nicht unumstritten.

Die Wandlungsphasen werden auch als Funktionskreise, Meridiane, Orbis, klassische Organe oder Speicher-Organe genannt.

Der Funktionalbezug der Elemente im "5-Wandlungsphasen-Modell" untereinander wird durch das folgende Pentagramm beschrieben:

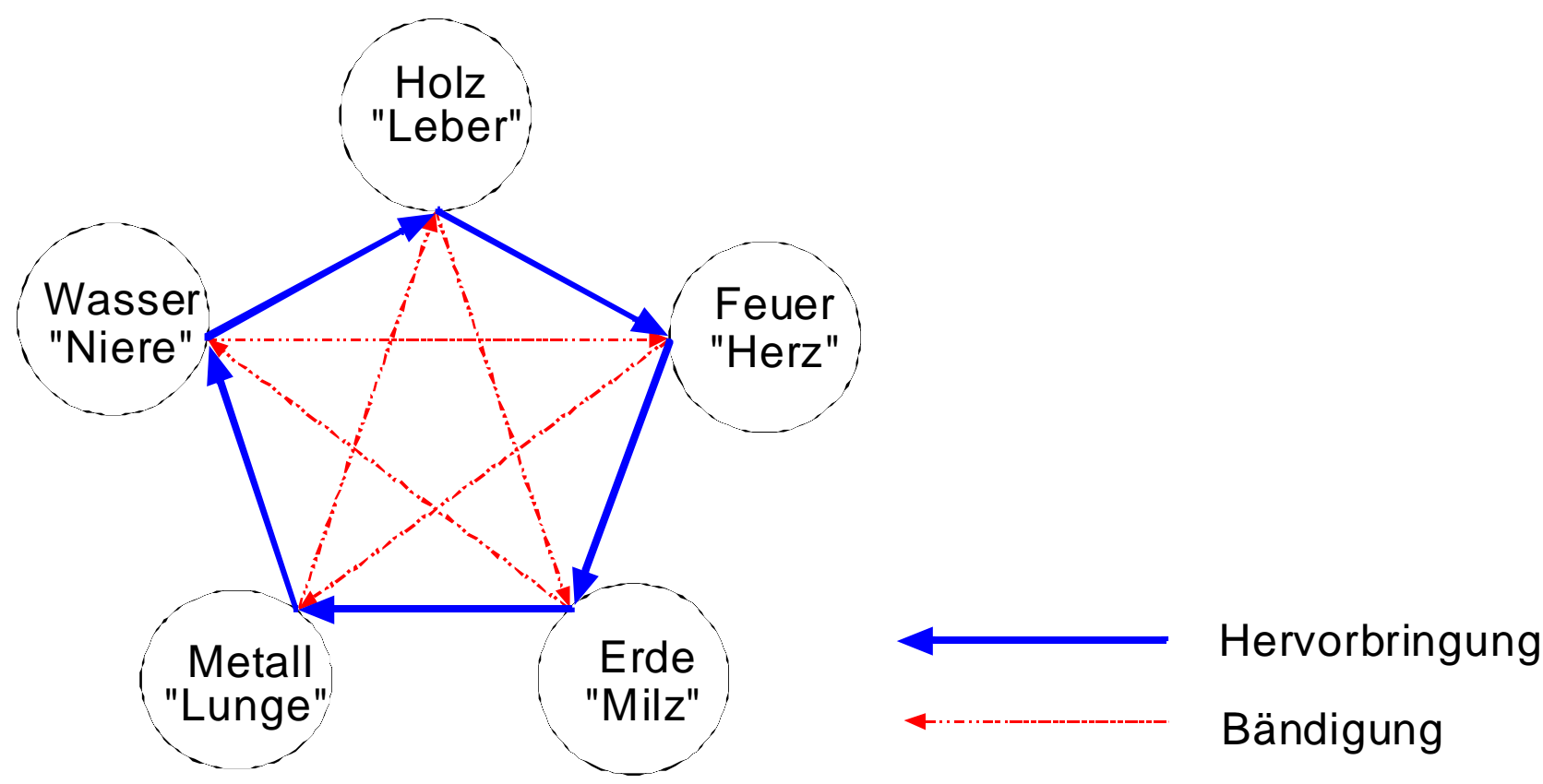

Die Funktionskreise werden in direkter Reihenfolge beeinflußt, d.h. der direkte Vorgänger fördert, veranlaßt oder erschafft den betrachteten Orbis. Der indirekte Vorgänger dagegen hemmt oder bremst den Funktionskreis.

Im gesunden Organismus besteht ein Fließgleichgewicht zwischen den Funktionskreisen, eine Störung diese Fließgleichgewichtes äußert sich als Krankheit.

Zur Therapie werden sowohl die Einflüsse der Hervorbringungs- Reihenfolge, als auch die der Bändigungsreihenfolge benutzt. 
Die Zuordnung einiger therapeutischer und theoretischer Begriffe $\mathrm{zu}$ den Funktionskreisen ist in der nachfolgenden Tabelle beschrieben.

\begin{tabular}{|l|l|l|l|l|l|}
\hline Element & Holz & Feuer & Erde & Metall & Wasser \\
\hline Meridian & $\begin{array}{l}\text { Gallenblase } \\
\text { Leber }\end{array}$ & $\begin{array}{l}\text { Herz, Dünndarm, } \\
\text { Herzkreislauf, } \\
\text { Dreifach- } \\
\text { Erwärmer }\end{array}$ & $\begin{array}{l}\text { Magen, } \\
\text { Milz }\end{array}$ & $\begin{array}{l}\text { Lunge, } \\
\text { Dickdarm }\end{array}$ & Blase, Niere \\
\hline Jahreszeit & Frühling & Sommer & $\begin{array}{l}\text { Spätsomm } \\
\text { er }\end{array}$ & Herbst & Winter \\
\hline Farbe & grün & rot & gelb-braun & weiß & $\begin{array}{l}\text { blau- } \\
\text { schwarz }\end{array}$ \\
\hline Sinnesorgan & Augen & Zunge & Mund & Nase & Ohr \\
\hline Sinn & Sicht & Sprache & $\begin{array}{l}\text { Geschmac } \\
\text { k }\end{array}$ & Geruch & Gehör \\
\hline Körpergewebe & $\begin{array}{l}\text { Muskeln, } \\
\text { Sehnen, } \\
\text { Gelenke }\end{array}$ & Blutgefäße & Fleisch & Haut & Knochen \\
\hline $\begin{array}{l}\text { Körperflüssigk } \\
\text { eit }\end{array}$ & Tränen & Schweiß & Speichel & Schleim & Urin \\
\hline Emotion & Ärger, Zorn & Freude & Mitgefühl & $\begin{array}{l}\text { Trauer, } \\
\text { Kummer }\end{array}$ & $\begin{array}{l}\text { Angst, } \\
\text { Furcht }\end{array}$ \\
\hline Geschmack & sauer & bitter & süss & $\begin{array}{l}\text { scharf- } \\
\text { würzig }\end{array}$ & salzig \\
\hline
\end{tabular}

\subsection{Das Qi und Wie}

Das dritte wichtige Konzept der traditionellen chinesischen Medizin ist das des Qui.

Das Qi ist ein recht umfassender Begriff, er läßt sich u.a. mit den Worten "Urkraft", "Atem", "Energie" beschreiben und ähnelt dem mittelalterlichen "Lebens-Äther"-Begriff. Das Qi wird auf alle dynamischen Prozesse angewandt, es ist ständig in Bewegung und praktisch überall vorhanden.

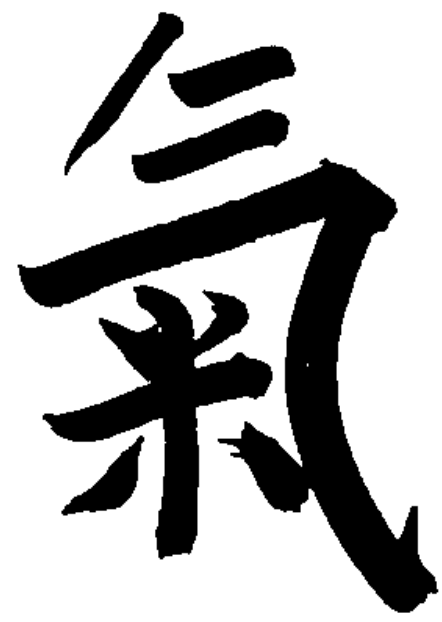


So gibt es ein Ursprungs-Qi, ein Nahrungs-Qi, ein Abwehr-Qi...

Der geordnete Fluß des Qi wird als Voraussetzung für die Gesundheit verstanden, Krankheiten resultieren aus einer Störung des Qi-Flusses. Der Qui-Fluß ist auch mental und emotional beeinflußbar. Auf dieser Basis arbeitende Therapiemethoden sind z.B. das Qi Gong (Qi-Übungen), welches mit dem Autogenen Training und der anthroposophischen Heileurythmie verwandt ist.

Das Qi hat keinen direkten Gegenspieler, wird aber durch das Wei ergänzt (vgl. Yang/Yin). Wei steht für die ordnende, abgrenzende Wehrenergie, das "Blut" im traditionell-chinesischen Sinn.

\subsection{Das Theoriebild}

Für die Diagnose und Therapie stehen alle drei (vier mit der klassischen weslichen Medizin) Therapieprinzipien als Werkzeuge zu Verfügung. Daneben existiert noch -ohne theoretischen Überbau- die "Daoistische Drogenkunde", vergleichbar mit den "volkstümlichen" Heilmitteln in Westeuropa.

Die genannten Theoriebilder werden noch durch weitere Begriffe ("Wärme") ergänzt und gemeinsam zur Diagnose und Therapie eingesetzt. Es existieren auch spezielle Therorien, die versuchen, die Konzepte in ein umfassendes Gesamtbild zu überführen. Weil aber absolute, geschlossene, endgültige Kategorien den fernöstlichen Denkprinzipen widersprechen, sind diese nicht so stark verbreitet.

Die bei der TCM benutzte Zustandsbeschreibung ist mehrdimensional und ohne genauere Kenntnisse oder entsprechendes Training nur schwer zu durchschauen. Welche der Theorien in welchem Umfange im konkreten Falle eingesetzt werden, entscheidet der Arzt.

Die Diagnose baut immer auf aktuellen Fließzuständen auf, ist ursachenorientiert: gleiche Symptome können verschiedene Ursachen haben und werden auch verschieden behandelt. Sie ist damit nicht verabsolutier- und/oder abstrahierbar.

Für statistische Untersuchungen zur Effektivität der Therapie ist es wegen der sehr ins Detail gehenden Diagnose sehr schwierig, eine Patientenbasis mit vergleichbarer/ gleicher Diagnose zu finden. Dies ist aber ein Problem aller alternativ-ganzheitlichen Therapiemethoden.

\section{Die Pharmakotherapie der TCM}

Die Pharmakotherapie der TCM beruht schwerpunktmäßig auf der Applikation von individuellen Drogenzubereitungen, seltener auf klassischen, konfektionierten Mischungen von Drogen.

Der enge Diagnosebezug führt dazu, daß mit einer Änderung des Allgemeinzustandes des Patienten durch wirksame Arzneimitteltherapie auch eine Rezepturänderung erfolgen muß. Damit können Fertigpräparate trotz der besseren Handhabbarkeit nur in untergeordnetem Maße eingesetzt werden. 


\subsection{Verwendete Materialien}

Die verwendeten Materialen sind pflanzlicher, tierischer oder mineralischer Natur und werden sowohl unverarbeitet als auch in vorbehandelter Form eingesetzt. Der größte Teil - ca. 80 \%- der Drogen ist pflanzlichen Ursprunges.

\subsection{Aufbereitungsformen}

Neben verschiedenen Trockungsmethoden (schnell/langsam, sonnig/schattig) sind je nach Droge noch verschiedene andere Vorbehandlungsverfahren in Gebrauch, die beispielsweise zu einer Entfernung giftiger Begleitstoffe führen und damit erst einen Gebrauch der Droge ermöglichen. Durch verschiedene Vorbehandlungsverfahren entstehen aus dem gleichen Ausgangsmaterial unterschiedliche Drogen mit unterschiedlichen Indikationen.

$\mathrm{Zu}$ den Aufbereitungsverfahren zählen die Reinigungsverfahren (Waschen, Auslaugen, Auslesen), Zerkleinerungsverfahren (trocken schneiden, feucht schneiden, zerreiben) und traditionelle Vorbehandlungsverfahren (Röst-, Brenn, Wasch- und Extraktionsverfahren).

\subsection{Zubereitung}

Die Drogen werden meist in Form eines wässrigen Auszuges oder Dekoktes verabreicht. Ethanol wird nur in einem Drogenvorbehandlungsverfahren eingesetzt, als Auszugsmittel wird Ethanol aufgrund seiner wirkstoffverändernden Eigenschaften und seiner Eigenwirkung abgelehnt.

Das Dekokt ist frisch herzustellen, die verwendete Menge an Rohdrogen ist recht hoch (z.B. soll das Wasser etwa $2 \mathrm{~cm}$ über den Drogen stehen). Drogengemische werden zur erschöpfenden Extraktion im allgemeinen zweimal ausgekocht.

Die Zubereitung erfolgt üblicherweise in Ton- oder emaillierten Gefäßen, nicht aber in Metalltöpfen (Abgabe von Metall-lonen). Auch hier sind verschiedene Sondervorschriften möglich, auf die in den einzenen Monographien des Arzneibuches hingewiesen wird.

\subsection{Drogeneigenschaften}

Die Eigenschaften der Drogen werden nach Geschmacksrichtung, Temperatur, Funktionskreisbezug, Wirkstärke, Wirkrichtung und Indikation beschrieben, so daß eine Zuordnung zum Krankheitsbild entsprechend der TCM-Theorie möglich ist. Zudem ist eine Beschreibung von beeinflußbaren Krankheiten mit entsprechender Ursache (veranlassende Funktionalstörung) verfügbar.

\subsection{Drogenbeurteilung}

Die Beurteilung der Drogen kann anhand der Vorschriften des Chinesischen AB vorgenommen werden. In diesem Werk aufgeführte Prüfmöglichkeiten entsprechen den allgemein bekannten Verfahren zur Drogenprüfung wie z.B. die Mikroskopische Prüfung, 
Bestimmung von Wassergehalt, Ätherischem Öl, pH-Wert, Schwermetallgehalt.

$\mathrm{Zu}$ beachten ist, daß mit den uns verfügbaren Methoden eine Idenditätsprüfung nur bei Rohdrogen und sehr eingeschränkt bei Drogenmischungen möglich ist, eine Prüfung fertiger Extrakte oder Zubereitungen ist mit den z.Zt. verfügbaren Materialien und Vorschriften nicht möglich.

\section{Praktische Hinweise}

\subsection{Fertigarzneimittel}

Fertigarzneimittel können recht leicht auf Plausibilität nach TCM geprüft werden:

Die Indikation sollte in den Begriffen der TCM angegeben sein und im Produkt sollte kein Alkohol enthalten sein.

Polypragmatische Präparate (mit mehr als 10 Bestandteilen) sind in ihrer Wirksamkeit qualitativ und quantitativ nur noch schwer einschätzbar und kritisch zu betrachten.

Übersetzungen der ursprünglichen Indikation ins Deutsche sind mangels entsprechender Begriffe im Deutschen sinnentstellend und damit nicht praktikabel.

Zu alkoholischen Extrakten existieren in der TCM keine Erfahrungswerte, sie sind wie einheimische Phytopharmaka mit neuen Drogen zu betrachten ([U+A7]49 AMG):

Für die alkoholischen Extrakte existiert weder eine therapeutische Tradition, noch ist das Spektrum der Inhaltsstoffe bekannt oder erprobt. Die Wirksamkeit und Unbedenklichkeit muß folglich durch den Inverkehrbringer nachgewiesen werden. Eine Zulassung/Registrierung als "traditionell angewandtes Mittel" ist damit weder formell, noch inhaltlich berechtigt. Alkoholische Extrakte sind folglich ganz normale, zulassungspflichtige Arzneimittel.

Derartige Zubereitungen sind aber auf dem Markt, insbesondere im Internet und im Versandhandel verfügbar. Hier sollte die Herkunft, Qualität und Sinnhaftigkeit besonders genau geprüft werden!

\section{Drogenvorbehandlung}

Bestimmte Drogen müssen vor dem Einsatz noch einem Vorbehandlungs- oder Aktivierungsverfahrten unterworfen werden (Rösten, Extrahieren). Durch unterschiedliche Verfahren können unterschiedliche Wirkungen erreicht werden. Manche Drogen werden erst durch diesen Schritt entgiftet. Besonders vorbehandelte Drogen erhalten auch eine von der Ausgangsdroge abweichende Bezeichnung.

\section{Rezepturgliederung}

Um bei der Vielzahl von Drogen noch die Wirkrichtung unterscheiden zu können, werden Drogen innerhalb einer Rezeptur in Herrscher- (Hauptwirkstoff), Minister(Nebenwirkstoff) Assistenten- und Botendrogen (Hilfswirkstoffe) unterschieden. Diese Einteilung ist mit der Wirkstoffeinteilung bei Phytopharmaka vergleichbar: auch hier wird zwischen Leitsubstanzen, weiteren wirksamen Bestandteilen und Begleitstoffen unterschieden.

Mit dieser Einteilung sind auch bei 8-12 Rezepturbestandteilen noch Wirkrichtungen abschätzbar. 


\section{Rezepturkennzeichnung}

Die Bestandteile einer Rezeptur können in verschiedener Form gekennzeichnet werden: in Chinesisch, in Latein, in der Pinyin-Umschrift und mit der deutschen Bezeichnung aus der deutschen Ausgabe des Arzneibuches der Chinesischen Medizin.

$\mathrm{Da}$ die Drogennamen in unterschiedlichen Regionen Chinas unterschiedlich ausgesprochen und einzelne Drogen auch unterschiedlich bezeichnet werden, sollte Verwechslungen vorgebeugt werden.

Dies geschieht am Einfachsten, indem jeder Bestandteil doppelt deklariert wird. Zu empfehlen ist die amtliche lateinische Bezeichnung und zusätzlich eine weitere Bezeichnung entweder in chinesisch oder in deutsch.

\section{6. benutzte Literatur}

Arzneibuch der Chinesischen Medizin, Deutscher Apotheker Verlag

Ihrig, Michael u. Syed Laik, Ali: Qualität von Drogen der Traditionellen chinesischen Medizin, Pharmazeutische Zeitung 6 (2001), S. $416 \mathrm{ff}$

Kapchuk, Ted J.: Das Große Buch der Chinesischen Medizin, Wilhelm Heyne Verlag München, 6. Auflage 1998

Porkert, M: Die Chinesische Medizin, Econ-Verlag Düsseldorf-Wien 1992 (nur noch antiquarisch verfügbar)

Reichling, Jürgen: Arzneimittel der komplementären Medizin, Govi Verlag 2001

Shaw, Debbie et al: Traditionelle Arzneimittel und Nahrungsergänzungsmittel (5jährige toxikologische Studie), Zeitschrift für Phytotherapie 19 (1998), S.323-327

Waller, Frank: Phytotherapie der traditionellen chinesischen Medizin, Zeitschrift für Phytotherapie 19 (1998), S. 77-89

Wolter, G.-A.: China-Spiegel, Verlag E.S.Mittler \& Sohn Herford 1978

Stand: 30.03 .2001

Anschrift des Autors:

Dr. Detlef Klauck

Apothekerkammer Sachsen-Anhalt

Dr.-Eisenbart-Ring 2

39120 Magdeburg 